\title{
Visual scanning of line segments: Object superiority and its reversal
}

\author{
MARSHA WIDMAYER and DEAN G. PURCELL \\ Oakland University, Rochester, Michigan 48063
}

\begin{abstract}
The object superiority and object line effects were investigated with a visual scanning task. Scanning times for two target lines were ordered as follows from fastest to slowest: no context, unconnected-lines context (flat), object context (three-dimensional), and disorganized context (flat). These results substantiate previous work (Klein, 1978) that demonstrated a reversal of the object superiority effect under speed stress when the unconnected-lines context was compared with the object context. However, these results also demonstrate that this reversal does not generalize to comparisons between disorganized and object contexts.
\end{abstract}

Several studies investigating the effect of context on visual perception have reported greater accuracy for the identification of a briefly flashed line segment when it was part of a figure that appeared unitary and threedimensional than when the same line was part of a two-dimensional or less coherent figure (Weisstein \& Harris, 1974). This effect has been called the object superiority effect (OSE). Williams and Weisstein (1978) have also reported an object line effect (OLE), in which a line within a unitary and three-dimensional figure is easier to recognize than is that line by itself. Klein (1978) eliminated the OSE by requiring speeded identification responses from his subjects. Further, Klein's latency data showed a reversal of the OSE, with longer reaction times to identify a line in a three-dimensional object context than to identify a line in a two-dimensional unconnected-lines context.

With the findings of Klein (1978), Weisstein and Harris (1974), and Williams and Weisstein (1978) in mind, a study was run to determine if Klein's (1978) reaction time findings could be replicated using a serial scanning task. Additionally, a larger set of contexts was employed. The present study was designed to investigate both the OSE and the OLE. Accordingly, four conditions were employed using three contexts of Weisstein and Harris (1974) and target lines presented alone.

\section{METHOD}

\section{Subjects}

Twenty-four naive subjects participated in the experiment.

\section{Stimuli}

The stimuli were taken from Weisstein and Harris (1974) and consisted of their three-dimensional "object" context, a disorganized context that they derived from the object context, and their "unconnected-lines" context. For examples of

Requests for reprints should be addressed to Dean G. Purcell, Department of Psychology, Oakland University, Rochester, Michigan 48063. these contexts, see Weisstein and Harris $(1974$, p. 754, Figures $2 \mathrm{a}, 2 \mathrm{~d}$, and $2 \mathrm{f}$, respectively). The target lines appeared either in conjunction with these contexts or by themselves, for a total of four conditions. The targets were lines that differed primarily in terms of slope, one positive and one negative, and are depicted in Weisstein and Harris (1974, Figures 1c and 1d). For an example of how these targets appeared in the object context, see Weisstein and Harris (1974, Figures $1 \mathrm{~h}$ and 1i). These stimuli were used to construct four 30-item practice lists and four 50-item experiment lists. Each list was blocked in terms of context. Each target appeared $50 \%$ of the time in each list in a random order, with the constraint that the same target line should not appear more than four times in a row. Separate sheets of paper were used to display the stimuli for each of the four conditions. The stimuli were black-on-white line drawings. They were placed in columns and spaced $13 \mathrm{~mm}$ from center to center. The target lines were $4 \mathrm{~mm}$ long. The object context was $8 \mathrm{~mm}$ wide and $8 \mathrm{~mm}$ high. The disorganized context was $10 \mathrm{~mm}$ wide and $10 \mathrm{~mm}$ high. The lines context was $8 \mathrm{~mm}$ wide and $9 \mathrm{~mm}$ high.

\section{Procedure}

Subjects were tested twice on each of the four practice lists and twice on each of the four experiment lists. Order of list presentation was counterbalanced across subjects. The subjects' task was to visually scan down each column of stimuli and name, in order, the target lines that they saw. They responded "right" if the lower part of the line pointed toward the right and "left" if the lower part of the line pointed toward the left. Subjects were instructed to read down the column as quickly as possible without making errors. Errors were not recorded. Scanning time was recorded, with an electronic stopwatch, for each trial to the nearest $.01 \mathrm{sec}$. Timing was begun when a cover sheet was removed from the stimulus sheet and the experimenter simultaneously said "now." Timing was ended with the subjects' last response.

\section{RESULTS}

The average scanning times were $21.79,22.21,24.41$, and $27.35 \mathrm{sec}$, respectively, for the target line alone and the unconnected-lines, object, and disorganized contexts. A repeated-measures analysis of variance indicated a significant effect of context $[F(3,69)=41.95$, $\mathrm{p}<.001]$. A planned comparison between the contexts employed by Klein (1978) replicated his finding that the 
OSE is reversed under speed stress. That is, the object context was scanned more slowly than the lines context $[F(1,69)=15.7, p<.01]$. However, an OSE was found when comparison was made between the object context and the disorganized context $[\mathrm{F}(1,69)=27.84, \mathrm{p}<.01]$, indicating that reversal of the OSE under speed stress is not necessarily a general phenomenon. There was no evidence for an OLE effect in the present data, with the target lines alone being scanned significantly faster than targets in the object context $[\mathrm{F}(1,69)=16.53$, $\mathrm{p}<.01]$.

\section{DISCUSSION}

In the present study, scanning times were fastest for those targets that were not connected to a context (targets alone and targets in unconnected lines). Banks and Prinzmetal (1976) have shown that perceptual isolation speeds target identification, and perhaps the same process is operating in both Klein's (1978) and the present study. While an isolation hypothesis can explain the present difference between the object context and the unconnected-lines context, it does not explain previous findings of an OSE with them (Weisstein \& Harris, 1974;Williams \& Weisstein, 1978). This discrepancy may be resolvable by considering the fact that Weisstein and Harris (1974) and Williams and Weisstein (1978) impoverished the quality of their stimuli through visual masking, in the first case, and through energy-level adjustments, in the second. This was done to control target identification at between $50 \%$ and $80 \%$ cor- rect. It may be that target isolation cannot manifest itself under these conditions. That is, a target within the unconnectedlines context may simply not have as good an image quality as a target in the object context. Hence, the potential scanning advantage for targets in the unconnected-lines context is unrealizable. Of course, this argument presupposes that the object context, under impoverished viewing conditions, actually facilitates perception. Comparisons between the object context and the disorganized context are not confounded by target isolation, so the OSE is robust across the impoverished viewing conditions of Weisstein and Harris (1974) and the nonimpoverished viewing conditions of the present experiment.

\section{REFERENCES}

Banks, W. P., \& Prinzmetal, W. Configurational effects in visual information processing. Perception \& Psychophysics, 1976, 19, 361-367.

KLEIN, R. Visual detection of line segments: Two exceptions to the object superiority effect. Perception \& Psychophysics, 1978, 24, 237-242.

Weisstein, N., \& Harris, C. S. Visual detection of line segments: An object-superiority effect. Science, 1974, 186, 752 755.

Williams, A., \& Weisstein, N. Line segments are perceived better in a coherent context than alone: An object-line effect in visual perception. Memory \& Cognition, 1978, 6, 85-90.

(Received for publication March 22, 1982.) 